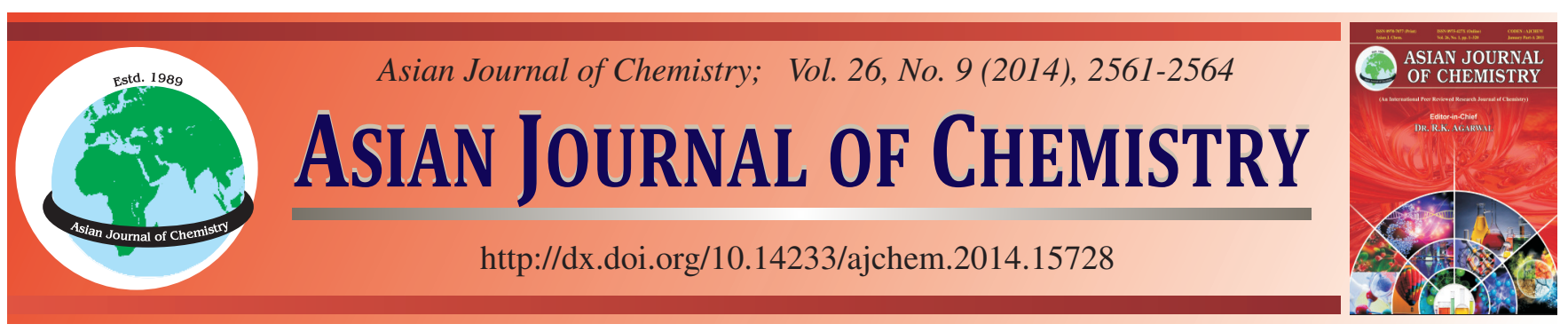

\title{
Rheological Study on Low Polymerization Degree Poly(vinyl chloride) Resin with JRG Constitutive Equation
}

\author{
YONG-QIANG DAI ${ }^{1}$, Li ZHANG ${ }^{2}$ and RI-GUANG JIN ${ }^{1, *}$
}

${ }^{1}$ State Key Laboratory of Chemical Resource Engineering, Beijing University of Chemical Technology, Beijing 100029, P.R. China ${ }^{2}$ Northwest Research Institute of Chemical Industry, Xi'an 710600, P.R. China

*Corresponding author: Fax: +86 10 64434850; Tel: +86 10 64434850; E-mail: jin.riguang@163.com; ken.workst@gmail.com

The low polymerization degree poly(vinyl chloride) resin has the property of low molecular weight and good flow ability. Through the study of three low polymerization degree poly(vinyl chloride) resins, we have found a relationship between rheological properties and molecular structure. We used the JRG constitutive equation to study the rheological properties of low polymerization degree poly(vinyl chloride). The three characteristic constants $\Delta \mathrm{E}^{\circ}, \alpha$ and $\beta$, which are closely related to the distribution of particles, granular property and molecular weight distribution, were determined.

Keywords: Poly(vinyl chloride), Rheological properties, JRG constitutive equation.

\section{INTRODUCTION}

Poly(vinyl chloride) (PVC) has been manufactured commercially for over 70 years. Since PVC was first studied at B.F. Goodrich, the PVC industry has developed rapidly. Poly(vinyl chloride) is one of the world's major polymers and a large amount of PVC is produced worldwide for its superior mechanical and physical properties ${ }^{1,2}$. There are many ways to synthesize $\mathrm{PVC}^{3}$. Not only PVC applications but also PVC types have been growing fast. In recent years, a variety of specialpurpose PVC resins have been synthesized. Low polymerization degree PVC is one of the major products. Low polymerization degree $\mathrm{PVC}$ is referred to $\mathrm{PVC}$ resins with a degree of polymerization of 600 or below. For their low molecular weight, low polymerization degree PVC resins are mainly used for making transparent films and plastic bottles. Although there are many papers on the study of rheological properties of $\mathrm{PVC}^{4-9}$, we have realized that there are few studies on the relationship between granular properties, molecular weight distribution and rheological properties of low polymerization degree PVC resins. Therefore some work on the rheological properties of low polymerization degree PVC resins will be described in this paper.

\section{EXPERIMENTAL}

Three different grades of low polymerization degree PVC resins (S-1, S-2 and J-1) were used in this study. S-1 and S-2 were with the degree of polymerization of 500 and 600 and purchased from Qilu Petrochemical Corp., Shandong Province, China. J-1 was with the degree of polymerization of 600 (use polyvinyl alcohol as dispersant) and purchased from Tianjin Chemical Corp., Tianjin, China.

Detection method: The apparent morphology of the low polymerization degree poly(vinyl chloride) resins were determined by mounting the samples and observing their features on a Cambridge S250 scanning electron microscope (SEM). The fractured specimen surfaces were coated with a thin layer (10-20 nm) of palladium. The coating was carried out by placing the specimen in a high vacuum evaporator and vaporizing the metal held in a heated tungsten basket. The viscosity at various shear rates was determined by using an Instron 3211 capillary rheometer. A single capillary die with an L/D ratio of 25 was used. The measurements were carried out in the shear rate range 1 to $10^{5} \mathrm{~s}^{-1}$ at three different temperatures: 165,175 and $185^{\circ} \mathrm{C}$.

\section{RESULTS AND DISCUSSION}

Observation of apparent morphology of PVC particles: Fig. 1 shows the SEM photographs of S-1 PVC particles. S-1 is a multi-cell structure resin. The particles are made of many primary particles and subparticles. Some particles are covered with a continuous smooth skin and others are covered with partial skins.

Fig. 2 shows the SEM photographs of S-2 PVC resin. S$2 \mathrm{PVC}$ resin is also a typical multi-cell structure resin. Some 


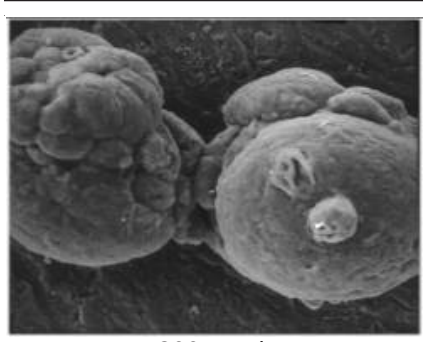

200 mesh

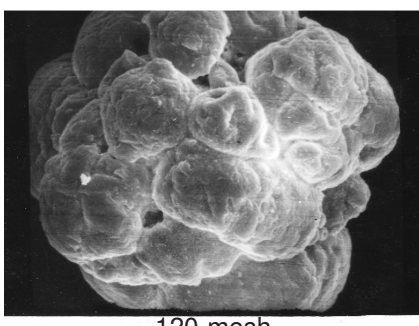

120 mesh

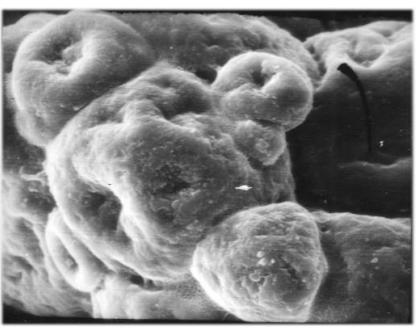

180 mesh

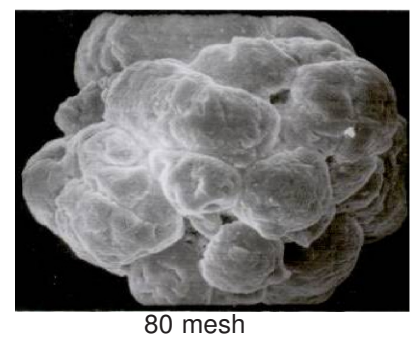

80 mesh

Fig. 1. SEM photographs of S-1 PVC particles of different meshes

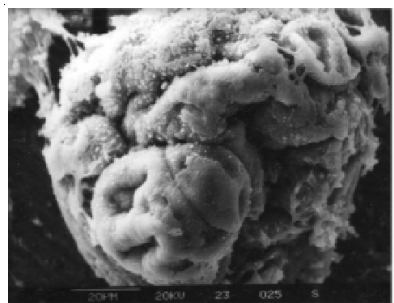

200 mesh

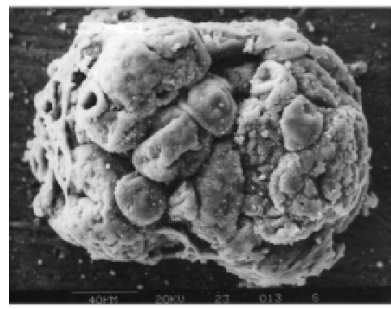

120 mesh

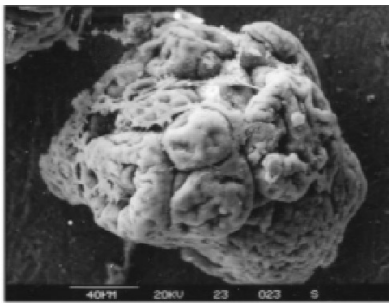

180 mesh

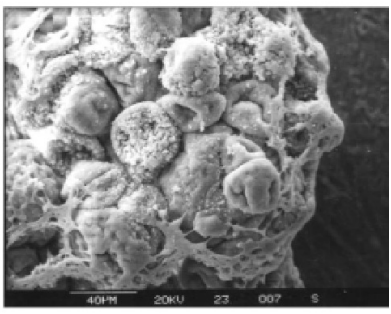

80 mesh
Fig. 2. SEM photographs of S-2 PVC particles of different meshes

particles are wholly made of primary particles with skin and some are made of all primary particles without skin. The most common structure is that the particles are made of primary particles and subparticles. The primary particles are skinless, while the skin of the subparticles is discontinuous. For the particles that don't have a continuous skin, the structure is not close is easy to break during processing.

Fig. 3 shows the SEM photographs of J-1 PVC resin. J-1 is a typical multi-cell structure resin as well. Some particles are covered with skin, which absorbs some primary particles and some are skinless. The surface of some particles with skin has some obturators.

JRG constitutive equation: The SEM photographs showed that the low polymerization degree PVC has different phase structures. We choose the JRG constitutive equation to describe the rheological properties of low polymerization degree PVC resins. The JRG constitutive equation is a generalized constitutive equation based on flow activation energy. It was first proposed by $\mathrm{Jin}^{10}$ to describe the relationship between the shear stress and shear rate accurately from the first Newtonian flow

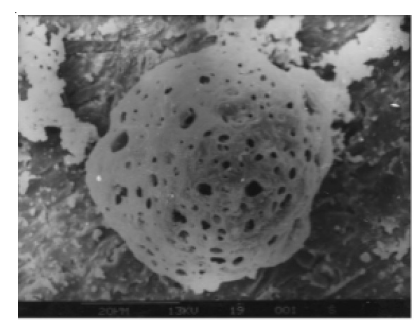

200 mesh

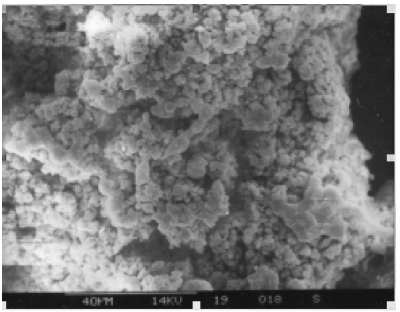

80 mesh

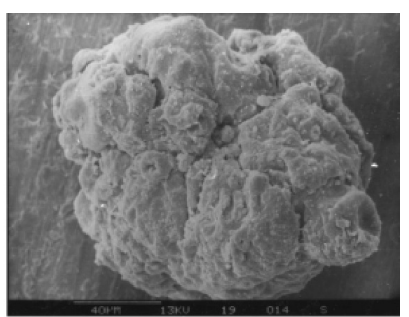

100 mesh

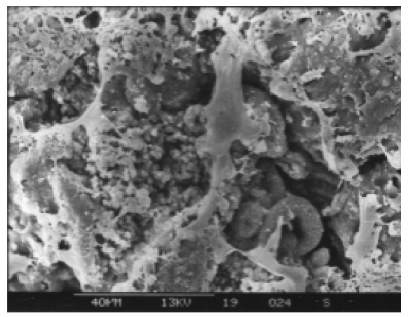

20 mesh
Fig. 3. SEM photographs of J-1 PVC particles of different meshes

region to the second Newtonian flow region. It was later named by Jiang $^{12}$ as the JRG constitutive equation based on flow activation energy model:

$$
\tau_{12}=\eta_{\infty}\left[\exp \left(\frac{\Delta \mathrm{E}_{\eta}^{0}}{\mathrm{RT}} \frac{1}{1+\alpha \dot{\gamma}^{\beta}}\right)\right] \dot{\gamma}_{12}
$$

The constitutive equation above eqn. 1 could not only describe the rheological behaviour in the first Newtonian region ( $\dot{\gamma} \rightarrow 0$ ), but also describe the rheological behaviour in the second Newtonian region $(\dot{\gamma} \rightarrow \infty)$. At the same time, this constitutive equation could fit can describe accurately both the pseudoplast flow accurately, if the parameter $(\alpha>0)$ and it could also fit the dilatant flow, if the parameter $(\alpha<0)$. It was shown from the flow curves (where?) that $\Delta \mathrm{E}^{\mathrm{o}}, \alpha$ and $\beta$ were the characteristic are material parameters of fluid which had nothing to do with independent of $\tau$ and $\dot{\gamma}$.

It can be seen from eqn. 1, that the flow activation energy $\Delta \mathrm{E}^{\mathrm{o}}$ corresponds to determine the temperature dependence of the viscosity of polymer melt, that is, the temperature dependence of viscosity on the temperature can be increased increases with the increase of increasing $\Delta \mathrm{E}^{\circ}$. The parameter $\alpha$ corresponds to determines the shear dependence of the viscosity of polymer melt. The shear dependence of viscosity increases with increasing $\alpha$. The viscosity decreases with the increase of shear rate $\dot{\gamma}$ when $\delta>0$ and the extent of the decrease of viscosity also increases with the increase of $\delta$. On the contrary, the viscosity increases with the increase of shear rate $\dot{\gamma}$ when $\alpha<0$. So the eqn. 1 can be used to describe the pseudoplast flow in which the melt viscosity decreases with the increase of shear rate or stress when $\alpha>0$ and it fits the dilatant flow in which the melt viscosity increases with the increase of shear rate or stress when $\alpha<0$. It is obvious that the equation fits the ideal Newtonian flow in which the viscosity is independent from the shear rate as $\alpha=0$. The parameter $\alpha$ is a characteristic constant of the polymer. $\operatorname{Jin}^{12-14}$ has studied 
HDPE, LDPE and blends of mPE and LDPE by using the JRG constitutive equation.

Sub-cluster parameters and rheological properties: In this work, we use the JRG constitutive equation to describe the rheological properties of high PVC resins. The rheological data are shown in Table-1.

\begin{tabular}{ccc|cc|ccc}
\multicolumn{8}{c}{ TABLE-1 } \\
\cline { 2 - 7 }$\dot{\gamma}_{\omega}$ & \multicolumn{1}{c}{ RHEOLOGICAL DATA FOR S-1 } \\
\cline { 2 - 7 } & $\log \tau$ & $\log \eta$ & $\log \tau$ & $\log \eta$ & $\log \tau$ & $\log \eta$ & \\
\hline 3.74 & 6.52 & 11.39 & 6.17 & 10.59 & 5.44 & 8.90 & 207.20 \\
12.48 & 6.6 & 10.37 & 6.33 & 9.75 & 5.8 & 8.53 & 153.15 \\
37.43 & 6.82 & 9.78 & 6.52 & 9.09 & 6.03 & 7.96 & 151.58 \\
124.76 & 6.95 & 8.87 & 6.69 & 8.28 & 6.31 & 7.40 & 122.47 \\
374.28 & 7.03 & 7.96 & 6.81 & 7.45 & 6.57 & 6.90 & 88.41 \\
1247.6 & 7.08 & 6.87 & 6.89 & 6.43 & 6.73 & 6.06 & 67.62 \\
\hline
\end{tabular}

By using JRG constitutive equation, we can get the equation $\mathrm{E} \eta=\mathrm{E}^{\mathrm{o}}\left[\frac{1}{\left(1+\alpha \dot{\gamma}^{\beta}\right.}\right]$. According to the linear slope (the plot is linear, not the slope) and intercept, we can obtain the values of $\mathrm{E}^{\mathrm{o}}$ and $\alpha$. The plots are as given in Figs. 4-6.

\begin{tabular}{|c|c|c|c|c|c|c|c|}
\hline \multicolumn{8}{|c|}{$\begin{array}{c}\text { TABLE-2 } \\
\text { RHEOLOGICAL DATA FOR S-2 }\end{array}$} \\
\hline \multirow{2}{*}{$\dot{\gamma}_{\omega}$} & \multicolumn{2}{|c|}{$165^{\circ} \mathrm{C}$} & \multicolumn{2}{|c|}{$175^{\circ} \mathrm{C}$} & \multicolumn{2}{|c|}{$185^{\circ} \mathrm{C}$} & \multirow{2}{*}{$\mathrm{E \eta}(\mathrm{kJ})$} \\
\hline & $\log \tau$ & $\log \eta$ & $\log \tau$ & $\log \eta$ & $\log \tau$ & $\log \eta$ & \\
\hline 3.74 & 6.45 & 11.23 & 5.92 & 10.01 & 5.45 & 8.93 & 191.98 \\
\hline 12.48 & 6.61 & 10.39 & 6.47 & 10.07 & 5.76 & 8.44 & 161.88 \\
\hline 37.43 & 6.92 & 10.01 & 6.73 & 9.57 & 6.21 & 8.37 & 136.36 \\
\hline 124.76 & 7.09 & 9.20 & 6.99 & 8.97 & 6.56 & 7.98 & 101.32 \\
\hline 374.28 & 7.18 & 8.30 & 7.12 & 8.17 & 6.74 & 7.29 & 83.80 \\
\hline 1247.6 & 7.29 & 7.20 & 7.18 & 7.10 & 6.85 & 6.34 & 71.34 \\
\hline
\end{tabular}

\begin{tabular}{ccc|cc|ccc}
\multicolumn{8}{c}{ TABLE-3 } \\
\hline \multirow{2}{*}{$\dot{\gamma}_{\omega}$} & $165^{\circ} \mathrm{C}$ & \multicolumn{2}{c}{$175^{\circ} \mathrm{C}$} & \multicolumn{2}{c}{$185^{\circ} \mathrm{C}$} & \\
\cline { 2 - 7 } & $\log \tau$ & $\log \eta$ & $\log \tau$ & $\log \eta$ & $\log \tau$ & $\log \eta$ & \\
\hline 3.74 & 6.86 & 12.17 & 6.48 & 11.30 & 6.28 & 10.84 & 111.22 \\
12.48 & 6.99 & 11.27 & 6.76 & 10.74 & 6.48 & 10.09 & 98.34 \\
37.43 & 7.06 & 10.33 & 6.92 & 10.01 & 6.64 & 9.36 & 80.73 \\
124.76 & 7.12 & 9.27 & 6.99 & 8.97 & 6.84 & 8.62 & 54.20 \\
374.28 & 7.16 & 8.26 & 7.04 & 7.98 & 6.96 & 7.80 & 38.44 \\
1247.6 & 7.19 & 7.12 & 7.07 & 6.85 & 7.02 & 6.73 & 32.62 \\
\hline
\end{tabular}

From Tables 1-3, we can see that the viscosity of S-1, S-2 and $\mathrm{J}-1$ decreases with the increase of the shear rate. It is suggested that the low polymerization degree PVC resins are pseudoplastic. From Figs. 4-6 and Table-4, we can see that the values of $\beta$ for the three kinds of low polymerization degree PVC resins (S-1, S-2 and J-1) are about the same. The values of $\mathrm{E}^{0}$ for $\mathrm{S}-1$ and $\mathrm{S}-2$ are, respectively 263.9 and $235.4 \mathrm{KJ}$, while the value of $E^{0}$ for $J-1$ is $204.5 \mathrm{KJ}$. The values of $\alpha$ for S-1 and S-2 are much lower than that for J-1.

The parameter $\alpha$ reflects the particles broken energy. The larger the value of $\alpha$ is the easier the particle is to break. From our previous work, we can see that there are a lot of black areas in the particles of S-2 PVC resin. There are fewer black areas in S-1 than in S-2, while there are only a few black areas in J-1. J-1 PVC resin particles have narrow molecular weight

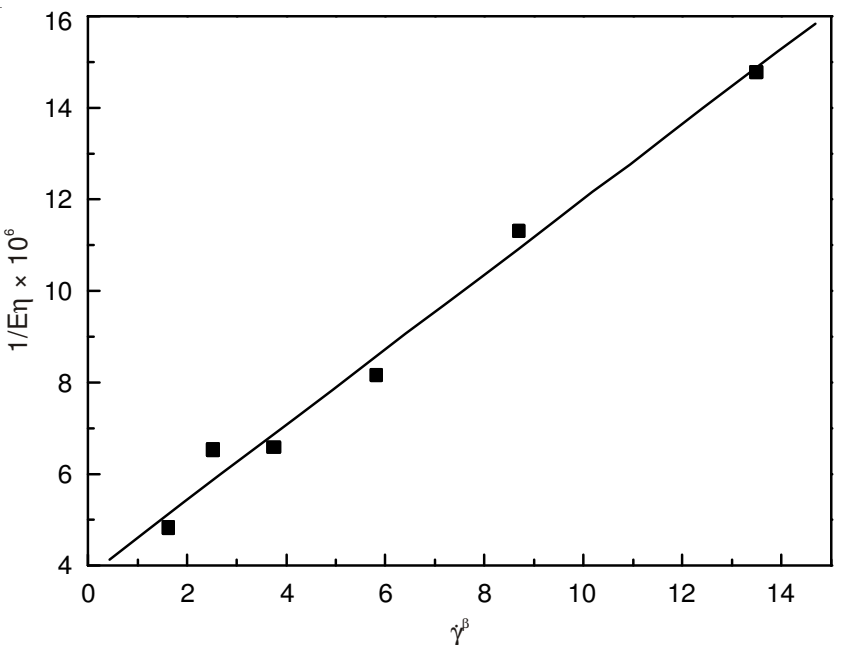

Fig. 4. Plot of $1 / \mathrm{E} \eta$ versus $\dot{\gamma}^{\beta}$ for $\mathrm{S}-1$

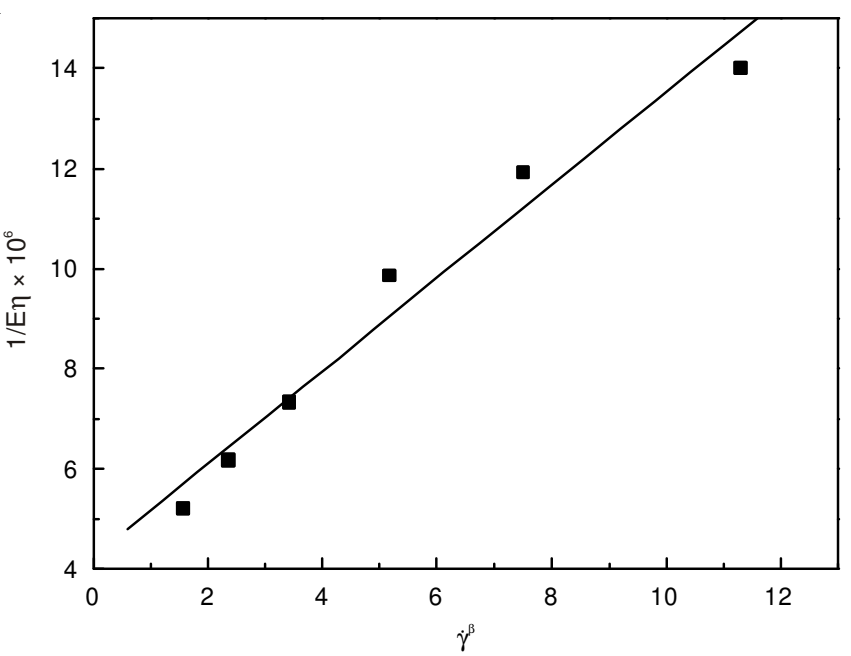

Fig. 5. Plot of 1/En versus $\dot{\gamma}^{\beta}$ for S-2

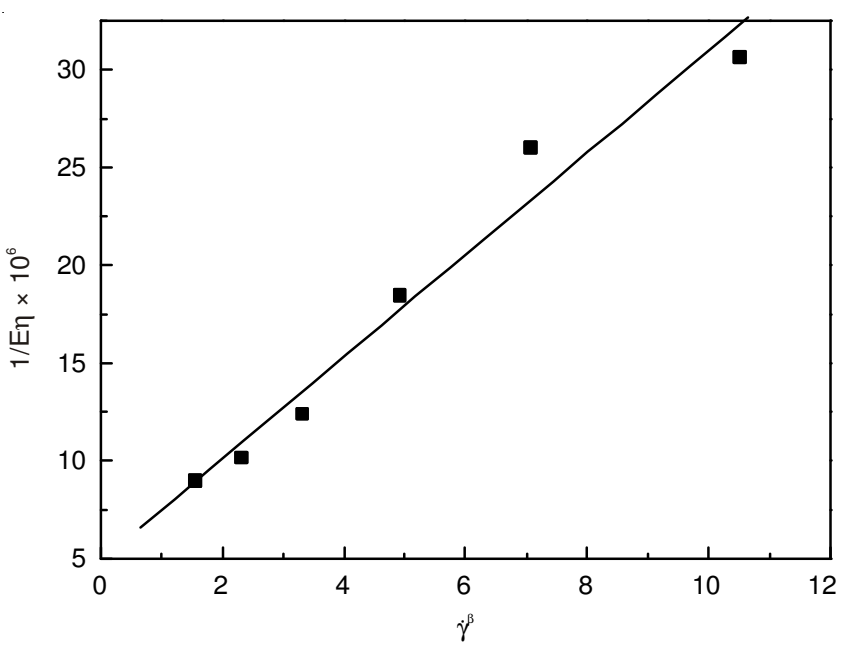

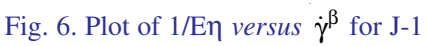

distribution, while S-2 PVC resin particles have wide molecular weight distribution. J-1 PVC resin particles are loose, uniform and easy to shatter. So the J-1 particles need less energy to break. In terms of the parameters of the JRG constitutive 
TABLE-4

PARAMETERS OF JRG CONSTITUTIVE EQUATION FOR LOW POLYMERIZATION DEGREE PVC RESINS

\begin{tabular}{ccccc}
\hline & $\mathrm{E}^{\mathrm{o}}(\mathrm{KJ})$ & $\alpha$ & $\beta$ & $\overline{\mathrm{M}}_{\mathrm{W}} / \overline{\mathrm{M}}_{\mathrm{N}}$ \\
\hline $\mathrm{S}-1$ & 263.9 & 0.217 & 0.365 & 2.74 \\
$\mathrm{~S}-2$ & 235.4 & 0.219 & 0.340 & 2.54 \\
$\mathrm{~J}-1$ & 204.5 & 0.533 & 0.330 & 2.01 \\
\hline
\end{tabular}

equation, the larger the value of $\alpha$ is, the less energy the particles need to break. And the larger the value of $\alpha$ is, the more sensitive the system is to shear.

The parameter $\beta$ reflects the melt non-Newtonian behaviour and it is related to the material structure. The larger the value of $\beta$ is, the smaller the non-Newtonian index of the melt is. From Figs. 1-3, we can see that the three kinds of low polymerization degree PVC resins have similar structures. All three kinds of particles have a multi-cellular structure, mainly made of primary particles and sub particles. So they have similar $\beta$ values. The values of $\beta$ are slightly different because the three kinds of low polymerization degree PVC resins have different molecular weights. The higher the molecular weight of the PVC resin is, the more non-Newtonian and viscoelastic the melt is. In terms of the JRG equation, this means a smaller $\beta$. J-1 has the highest molecular weight and S-1 has the smallest molecular weight, corresponding to the highest value of $\beta$ for $\mathrm{S}-1$ and the smallest value of $\beta$ for $\mathrm{J}-1$.

The parameter $\mathrm{E}^{0}$ reflects the energy of particle breakage under zero shears. From previous work ${ }^{14}$, we can see there are a large number of black areas in the SEM photographs of S-1 and $\mathrm{S}-2$, while there are fewer black areas in J-1 than in S-1 and S-2 and the black areas in J-1 spread uniformly. J-1 has fewer high-molecular-weight particles which are hard to break and the particles of J-1 have a loose structure. So the value of $\mathrm{E}^{0}$ of $\mathrm{J}-1$ is $204.5 \mathrm{KJ}$, which is smaller than those of S-1 and $\mathrm{S}-2$. The value of $\mathrm{E}^{0}$ of $\mathrm{S}-1$ is $263.9 \mathrm{KJ}$, largest in the three kinds of low polymerization degree PVC reins. The particles of S-1 need much more energy to break because the particles of $\mathrm{S}-1$ have a continuous skin and the skin is relatively smooth and the value of $\mathrm{E}^{0}$ is the largest. From Tables 1-3, we can see that the viscosity of $\mathrm{J}-1$ decreases with increasing temperature more slowly than that of S-1 and S-2.

Thus, it is found that the JRG constitutive equation can be used to describe the rheological properties of low polymerization degree PVC resins.

\section{Conclusion}

Low polymerization degree PVC resins have a multi-cell structure and the particles are mainly made of primary particles and sub particles. Through the study on the relationship of sub-cluster parameters $\mathrm{E}^{0}, \alpha, \beta$ and the rheological properties of PVC resins, we have found that the sub-cluster parameters can reflect the rheological properties of the low polymerization degree PVC resins. It is further proved that the JRG constitutive equation has universal and guiding significance in dealing with rheological problems

\section{REFERENCES}

1. M. Kaufman, The History of PVC, Maclaren \& Sons, London (1969).

2. K. Schmieder and K. Wolf, Kolloid-Z, 127, 65 (1952).

3. K. Endo, Prog. Polym. Sci., 27, 2021 (2002).

4. N. Nakajima and E.R. Harrell, J. Colloid. Interf. Sci.., 238, 105 (2001).

5. M.G. Rasteiro, A. Tomás, L. Ferreira and S. Figueiredo, J. Appl. Polym. Sci., 112, 2809 (2009).

6. N. Sombatsompop and S. Thongsang, J. Appl. Polym. Sci., 82, 2478 (2001).

7. G.G. Liang, W.D. Cook, H.J. Sautereau and A. Tcharkhtchi, Polymer, 50, 2635 (2009).

8. E.G. Barroso, F.M. Duarte, M. Couto and J. M. Maia, J. Appl. Polym. Sci., 109, 664 (2008).

9. A. Mousa, N.A. Halim and A. Al-Robaidi, Polym.-Plastics Technol. Eng., 45, 513 (2006).

10. R.J. Jin, The Progress of Rheology, IV, South China University of Science and Technology Press, Guangzhou, p. 17 (1933).

11. T.Q. Jiang, Chemical Engineering Rheology, East China Institute of Chemical Technology Publishing Company, Shanghai, China, p. 124 (2004).

12. R.J. Jin, Polymer Rheology and Its Processing, Chemical Industry Press, Beijing, China, p. 43 (1986).

13. R.G. Jin, Advances in Rheology(IV), Huazhong University of Science and Technology Publishing Company.Guangzhou, China, p. 17 (1993).

14. R.G. Jin and H.Q. Lin, Proceedings of XI International Congress on Rheology, Brussels, Belgium, p. 159 (1992). 